\title{
Mise en proximité et politique. Les «Carnets de campagne » du Monde (19 mars - 2 juin 2002)
}

\section{Christiane Restier-Melleray}

\section{(2) OpenEdition}

\section{Journals}

Édition électronique

URL : https://journals.openedition.org/mots/142

DOI : $10.4000 /$ mots. 142

ISSN : 1960-6001

Éditeur

ENS Éditions

\section{Édition imprimée}

Date de publication : 1 mars 2005

Pagination : 59-72

ISBN : 2-84788-077-1

ISSN : 0243-6450

\section{Référence électronique}

Christiane Restier-Melleray, "Mise en proximité et politique. Les «Carnets de campagne » du Monde (19 mars - 2 juin 2002)», Mots. Les langages du politique [En ligne], 77 | 2005, mis en ligne le 31 janvier 2008, consulté le 23 avril 2022. URL : http://journals.openedition.org/mots/142 ; DOI : https://doi.org/ $10.4000 /$ mots. 142

\section{(C) ENS Éditions}




\section{Mise en proximité et politique. Les «Carnets de campagne " du Monde (19 mars - 2 juin 2002)}

Nous examinerons la mise en scène journalistique du rapport profane à la sphère traditionnellement lointaine du politique, mise en scène construite par l'intermédiaire d'un genre ${ }^{1}$ : les "Carnets de campagne », publiés très régulièrement en dernière page du Monde lors des campagnes présidentielle et législative de 2002, au rythme à peu près respecté de cinq parutions hebdomadaires, du 19 mars au 2 juin (soit soixante-quatre Carnets²).

S'il est un exercice journalistique qui répond au critère de proximité, c'est bien le Carnet. Le journaliste est censé restituer les choses saisies sur le vif, il ne serait que le porteplume qui s'effacerait, voyant et entendant à la place de celui qui lira. Le genre postule des proximités emboitées : celle du lecteur et du journaliste, celle du journaliste et du terrain, toutes deux conditionnant une troisième proximité, celle du lecteur et du récit. Ce dispositif reprend la tradition de l'écho, mais revisitée par la pratique ethnographique dans une logique de feuilleton, puisque les Carnets se succèdent au fil du macroévènement dont ils rendent compte.

Sont ainsi livrées des choses censées être vues mais aussi - et surtout entendues. C'est au seul récit qui nous est délivré que nous nous intéresserons, récit qui prend d'autant plus d'importance que le journaliste responsable du reportage semble s'effacer derrière son objet, le Carnet reposant sur le présupposé de la transparence des faits sociaux ${ }^{3}$. On ne saurait trop souligner l'unité de ce dispositif textuel : tous les Carnets, qu'ils soient rédigés par des journalistes parisiens ou par des correspondants locaux, proposent la

1. Défini tant du point de vue du dispositif textuel que de celui de sa nature communicationnelle. Sur le recours à cette terminologie: P. Charaudeau, D. Maingueneau, 2002, Dictionnaire d'analyse du discours, Paris, Le Seuil, p. 277 et suiv.; D. Maingueneau, 1998, Analyser les textes de communication, Paris, Dunod, p. 45 et suiv.; P. Charaudeau, 1997, Le discours d'information médiatique. La construction du miroir social, Nathan-INA, p. 129 et suiv.; F. Jost, 1997, «La promesse des genres ", Réseaux, $\mathrm{n}^{\circ} 81$.

2. Comportant chacun environ 750 mots.

3. Consacrant une «rhétorique de l'amont», selon la formule de D. Ruellan, 1993, Le professionnalisme du flou, Presses universitaires de Grenoble.

Université Bordeaux 4, CERVL-CNRS, c.restier@wanadoo.fr 
même mise en récit. L'exercice mobilise plusieurs déclinaisons et plusieurs niveaux de proximité : la connivence qui lie le lecteur au journaliste ${ }^{4}$, la posture compréhensive qui lie le journaliste à son terrain, et un dispositif textuel spécifique. Pour ce faire, le journaliste utilise une certaine rhétorique, a recours à des catégories sémantiques indigènes et use de procédés de construction et de mise en scène spécifiques.

Nous examinerons dans un premier temps la mise en œuvre de ces récits et préciserons ensuite quelle vision de la société y est privilégiée, quelle image du rapport profane au politique est proposée, avant d'analyser, dans une approche plus dynamique, quels sont les ressorts mobilisés pour établir la connivence avec le lecteur. Mais cette étude sera guidée par la recherche d'un éventuel changement de cadrage journalistique induit par la présence au deuxième tour de l'élection présidentielle du leader du Front national et l'éviction du candidat socialiste. Cette situation de quasi-expérimentation nous permettra de tester au fil de l'analyse la validité de nos observations dans le cadre de deux séquences : d'une part celle qui précède et, d'autre part, celle qui suit ce que les commentateurs ont nommé le «coup de tonnerre» du 21 avril.

\section{La proximité par la description}

Appréhender une campagne électorale du point de vue du quotidien des profanes débouche sur des mises en récit présentant les mêmes caractéristiques, tant avant qu'après le 21 avril.

\section{Donner à voir et à entendre}

Le Carnet repose sur une mise en forme spécifique 5 abolissant la distance. La technique consiste, dans la quasi-totalité des cas, à opérer un zoom sur un individu ou, au plus, sur un groupe, et à procéder ensuite à une montée en généralité. On présente le particulier dans sa singularité, on identifie ceux qui parlent (les patronymes étant très souvent évoqués dès le titre ${ }^{6}$ ). L'utilisation

4. L'existence présupposée d'un lecteur-modèle du Monde conditionne largement le contenu sémantique et lexicologique des textes.

5. Dont atteste le traitement informatique par une analyse lexicométrique (logiciel Lexico), mais aussi par une analyse propositionnelle de discours (logiciel Tropes).

6. Un procédé qui s'accentuera au fil des semaines et dont on sait qu'il n'est pas habituellement utilisé en sociologie, où l'anonymat est de règle. C'est ainsi que la première séquence s'ouvre sur le dialogue d'un couple d'octogénaires parisiens («Annie votera finalement Chevènement, et Jacques reste fidèle au PC») et se termine sur un autre dialogue ("Alain à sa fille : “J'ai l'impression d'être sur un bateau qui coule.” »). Mais, entretemps, nous avons pu dès le titre pénétrer dans des univers immédiatement accessibles grâce aux prénoms : "Hassen et Naïma vont 
des noms et prénoms7, l'ancrage géographique des récits (y compris dans la mise en forme de l'article accompagné d'une carte de France avec une flèche localisant le lieu d'investigation) créent la proximité et authentifient simultanément le récit. L'effet de réel en constitue le ressort ${ }^{8}$. Le recours au vocabulaire familier, aux préoccupations quotidiennes et aux analyses personnelles s'impose. On peut en juger en rappelant ce commentaire des résultats du 21 avril :

Mon grand-père vote Le Pen, certainement à cause de la guerre d'Algérie. Maman lui est fidèle depuis l'âge de 18 ans. Elle travaille dans un tribunal, et ce qu'elle voit et entend tous les jours doit compter pour beaucoup dans ses convictions. Mon père préfère la droite molle, comme dit maman... Par contre, mes petites sœurs, 11 et 15 ans, sont fans de Le Pen. Elles collectionnent ses posters... À cet âge-là, c'est normal qu'elles soient influencées par le vote de leur mère...

La quasi-totalité des Carnets des deux séquences se caractérise par une prise en charge du récit par les narrateurs. Loin d'être des reportages strictement descriptifs, ils sont structurés autour du développement d'un argumentaire instaurant avec le lecteur une proximité fondée sur la restitution d'échanges profanes censés s'exprimer en toute spontanéité : donner à voir la politique par le bas passe par la mise en scène de discussions formulées dans le langage de tous les jours et particulièrement animées. En attestent le recours élevé à des joncteurs d'opposition et une utilisation exceptionnelle de la modalisation de négation?.

Ajoutons que, dans l'ensemble des Carnets, les locuteurs s'engagent, expliquent ou critiquent, essaient de persuader. Mais ce qu'il faut bien considérer comme l'expression d'opinions politiques est présenté dans un langage apparemment non politique, celui de tous les jours, y compris dans la séquence postérieure au séisme du 21 avril, séquence pour laquelle les classes sémantiques mobilisées réfèrent pourtant à la politique et aux élections. La proximité avec les profanes implique la mise en récit d'univers sociaux spécifiques. Rien n’y est laissé au hasard : tout y fait sens, ainsi qu'on va le constater.

voter pour la première fois en France » (le 2 avril), "Claude et Sébastien, branchés, abstentionnistes et fiers de l'être » (le 3), "L'indignation de Jeanne et André, éleveurs de porcs bretons » (le 4), «Pierre et Bénédicte, marins pêcheurs, heureux de voter» (le 6), "Jacky et Marcel, agriculteurs, séduits par François Bayrou» (le 9), "Jacques, électeur frustré sur les forums électroniques» (le 10), "Jean-Pierre, pas militant, mais “accro” des joutes politiques » (le 13), «André, la passion de la chasse et de la défense de la nature» (le 17), "Jacques, Danièle et Richard mobilisent “discrètement” au Sénégal » (le 19), «Claude et Alain, préretraités et "amiantés” : à gauche, toute !» (le 20).

7. Précisons qu'on évoque aussi par leur prénom les femmes politiques (à l'exception de C. Boutin).

8. R. Barthes, 1968, Communications, $\mathrm{n}^{\circ} 11$.

9. Selon l'évaluation du logiciel Tropes : la modalisation d'intensité atteint pour l'ensemble des textes $35 \%$. 


\section{Le social, addition d'histoires de ceux qui "savent de quoi ils parlent»}

Pour faire toucher au lecteur du Monde des milieux qui lui sont largement étrangers, le journaliste est confronté au problème du traitement sociologique d'univers sociaux particuliers; il lui faut restituer sans déformer, respecter tout en faisant comprendre. Cependant, quelle que soit la prégnance de l'objectif d'authenticité, le journaliste ne rapporte pas de la même façon les faits et gestes d'un cadre supérieur, d'un ouvrier ou d'un SDF. Il semble que les chroniqueurs considèrent que seul un traitement différencié, construit de façon souvent stéréotypée, voire caricaturale, soit paradoxalement susceptible de faciliter la compréhension de comportements et opinions exotiques. S'agissant des milieux populaires, le misérabilisme n'est pas loin et, en tout état de cause, l'évocation des détails matériels de l'existence n'est pratiquement abordée que dans leur cas.

Ainsi on s'attarde sur Claude Boyer-Joly, 54 ans, et Alain Bodevin, 56 ans, préretraités et amiantés : métallos syndiqués à la CGT... «Tous deux ont commencé à travailler à 14 ans, le premier avec un CAP de chaudronnier, le second avec un CAP d'électricien... » On indique avec leurs propres mots qu'ils "ont dû faire des "heures sup à la pelle”, "y compris les nuits ou les week-ends", pour "pousser" leurs enfants le plus loin possible - la fille ainée de Claude est médecin, son fils professeur de lycée technique... Les deux filles d'Alain sont infirmière et attachée commerciale... » On fait toucher du doigt l'importance du vécu et du concret : "Les voici tous deux préretraités et "papies" - trois petitsenfants pour Claude, quatre pour Alain. L'un a fait construire une villa à $25 \mathrm{~km}$ du Havre et soigne amoureusement son jardin, l'autre habite toujours la "ville haute" mais possède un bout de terrain pour "faire le barbecue".»

C'est une profusion de détails et un recours systématique à la description des petites choses du quotidien qui caractérisent le traitement des catégories sociales dominées. Désignés par leur prénom, parlant de leurs problèmes les plus matériels, ces catégories «bossent», souffrent, ont des joies simples, des préoccupations matérielles et le disent dans un langage vernaculaire. La personnalisation - qui parfois flirte avec le voyeurisme - et l'évocation du quotidien caractérisent les deux séquences.

Le Carnet obéit donc non seulement aux contraintes littéraires de la recherche de l'effet de réel mais aussi aux canons du journalisme social : la description méticuleuse des conditions de vie, amarrant l'analyse, permet la compréhension. Mais, censée équivaloir à une restitution de l'ancrage social, elle donne seulement à voir du social individualisé et largement dépolitisé, dans la mesure où le journaliste brosse des Carnets s'inscrivant dans une campagne 
électorale sans accorder d'importance à la restitution des activités proprement politiques : le champ politique est désenclavé du champ social. C'est ainsi que les Carnets de la première séquence n'accordent que peu de place à l'actualité politique ${ }^{10}$, privilégiant les préoccupations du quotidien ${ }^{11}$. La politique semble indifférer, ce que confirme un francophile allemand : "Les politiciens établis sont coupés de ce qui tracasse les gens.» Le politique ne peut faire intrusion que s'il se déplace au devant des acteurs et dans le cadre de leurs activités non politiques. Ainsi, pour les agriculteurs Jacky et Marcel ayant accueilli F. Bayrou, «cette visite fera date. Jamais une telle personnalité n'avait mis les pieds dans leur ferme. Jamais, non plus, ils n'avaient vu un postulant à l'Élysée s'aventurer ainsi sur le terrain ».

Mettre en récit la vie des profanes revient à imposer par la pesanteur des expériences concrètes l'idée qu'aucun témoignage n'est fortuit ou gratuit. Mais c'est aussi postuler que ce ne peut être que sous l'angle des politiques publiques telles qu'elles sont vécues, par le bas, que la politique peut être appréhendée : c'est à la vie de tous les jours que les élus sont supposés devoir consacrer leur activité, et c'est in fine sur ce critère que l'ensemble des Carnets nous dit qu'ils doivent être jugés ${ }^{12}$. Le manque de politique apparait encore plus flagrant lorsque le quotidien de chacun est présumé être soumis à des décideurs évoluant dans un univers lointain. Tous ces Carnets s'inscrivent dans la cartographie de "l'espace des possibles rhétoriques» proposée par É. Neveu ${ }^{13}$ et l'on peut filer plus avant cette métaphore géographique : c'est bien une cartographie de l'espace social qui les sous-tend, ainsi que nous allons le montrer.

\section{La distance, clé du rapport au politique}

En effet, si la description réaliste cimente la proximité journaliste / interviewé / lecteur, une telle proximité a pour corollaire l'affirmation de la distance au

10. La moitié des Carnets de cette séquence n’aborde pas la politique en première classe sémantique.

11. Ainsi, les adhérents d'une crèche parisienne, où «l'ambiance générale est plutôt de gauche, selon le directeur (gaillard barbu qui parle tout en changeant la couche de la petite Iris) », sont présentés comme «des gens ordinaires, qui veulent participer à l'éducation de leurs enfants et être des acteurs de leur quartier», alors que leurs propos et leurs activités associatives attestent de leur indéniable compétence politique.

12. «Les privatisations, le retrait du service public, les décisions européennes, cela relève à Paris de débats théoriques, mais ici, c’est du vécu quotidien... »

13. É. Neveu, 1993, "Pages “Politique" », Mots, n 37, p.14-15 : «l'écriture se fait ici naturaliste, s'emploie à restituer les émotions, la chaleur du vécu... La rhétorique de l'asyndète, l'effet de réel du croquis font de ces instantanés des moments de pittoresque plus destinés à être savourés qu'analysés... » 
politique; ici encore «le proche ne fait sens que par rapport au distant» ${ }^{14}$. Nous envisagerons cette question en prenant successivement en compte les deux vagues de récits afin d'appréhender les éventuelles incidences du résultat du 21 avril sur la permanence de la mise en exergue de cette distance.

\section{L'avant 21 avril de la société civile}

L'observation de la première séquence atteste que les situations s'ordonnent très précisément en fonction du rapport des interviewés au politique. On pourrait d'ailleurs répertorier ces premiers textes à l'aune de la distance que les individus mis en scène entretiennent avec le jeu politique conventionnel. Ceux qui participent au jeu classique sont minoritaires, voire un peu ridiculisés ${ }^{15}$, tandis que l'éloignement du champ politique s'impose comme une évidence. La distance à l'égard du jeu politique conventionnel est largement mise en scène. Très... classiquement, si l'on ose dire, par les citoyens protestataires (Front national, CPNT, écologistes de la première heure), mais aussi par les membres de groupes socioprofessionnels qui, mettant en exergue leurs problèmes quotidiens, procèdent à une sévère condamnation des politiciens. À la frontière de ce premier cercle, on peut repérer des groupes, le plus souvent critiques, s'excluant du jeu partisan (abstentionnistes, associatifs, libres penseurs, chômeurs). Plus loin encore se trouvent les hors-jeu, sans-papiers, SDF, le cas extrême étant symbolisé par le dispositif de «l'Urne des sans voix» de Médecins du Monde à Bordeaux.

Les récits obéissent à une logique implacable qu'on pourrait présenter sous forme de syllogisme : la vraie vie est celle des gens d'en bas, il y a coupure entre les politiques et les gens d'en bas, donc les politiques sont coupés de la vraie vie. Dès lors, lorsqu'il décrit la vraie vie, le journaliste est crédité d'un brevet de proximité et d'authenticité qui n'est validé que par le postulat de la coupure des politiques d'avec cette réalité. En témoignent les propos peu charitables des sœurs de Goarec, présentées comme des modèles de civisme :

«Que de platitudes, de langue de bois et de démagogie !» s'exclame sœur Geneviève, «quelle indignité dans les attaques réciproques, et surtout, quelle soif incommensurable de pouvoir! La France a connu de grands personnages... Mais il y a désormais trop de malhonnêteté. Et cette façon de s'incruster ! Le personnel politique devrait être renouvelé. Il a perdu de vue l'objectif essentiel : le service de l’État.»

14. Selon la formule de R. Lefebvre, 2000, «Rhétorique de la proximité et “crise de la représentation" ", Cahiers lillois d'économie et de sociologie, n 35-36.

15. Tel ce cadre supérieur "accro des joutes politiques» ou ces octogénaires de Saint-Germaindes-Prés dont la mise en dialogue évoque un remake Sartre/Beauvoir. 
Et la journaliste A. Cojean de renchérir : «Ce n’est pas un péché véniel. Car les sœurs s’y connaissent en démocratie, fières du règlement de leur congrégation... ». La conclusion s’impose : "Elles aimeraient voter avec élan et plaisir. Ce sera pour une autre fois.»

La critique du politique constitue indéniablement le fond de carte des Carnets antérieurs au 21 avril $^{16}$, l'examen circonstancié de groupes considérés comme typiques le confirme. À ce titre, une place importante est accordée à ce qui est présenté par les journalistes comme une entité, les jeunes. Leur coupure avec la sphère politique est martelée et considérée comme une spécificité générationnelle ${ }^{17}$, ce que résume une fille d'agriculteurs :

Mais qu'est-ce qu'on me propose ? Voter pour le moins pire ? Voter pour ces vieux blasés et compromis qui vivent dans leur bulle et s'accrochent au pouvoir? Moi je voudrais me dire que nous, les jeunes qui arrivons, on va faire bouger les choses. Eh bien non. Les jeux se font entre vieux énarques qui ne me parlent pas de mon avenir. Déprimant!

\section{L'après 21 avril des espaces publics profanes}

L'examen des seconds Carnets permet de tester l'hypothèse de la permanence de ce cadrage. On peut tout d'abord constater que ces textes privilégient eux aussi les réactions d'individus ou de groupes restreints mais, dans ces groupes, on parle politique. Les personnages mis en récit ne se détournent pas, loin s'en faut, des activités citoyennes. L'évènement les amène au contraire à réfléchir à leurs pratiques, justifier ou réviser leurs opinions. Les proclamations de foi anarchisantes ne sont plus ici synonymes de désintérêt citoyen mais attestent de la participation à la communauté nationale. On peut être libertaire mais faire de l'animation sociale, être abstentionniste par principe mais donner de sa personne en animant un collectif antidrogue. Aussi n'est-il

16. On peut en juger par les contextes lexicaux des mots «politique» et «politicien » : «la politique pour nous, c'est de galérer à la mairie» / " dès qu'on leur parle de politique, les visages se font sarcastiques ou hostiles » / "la politique l'indiffère » / "il rejette lui aussi toute forme d'action politique, au nom de la modernité» / « le personnel politique devrait être renouvelé » / «ils étaient écœurés de la politique» / «les politiques se sont oubliés» / «les hommes politiques, dont la vertu n'a jamais été le courage» / " tu n'as qu'à devenir la copine d'un politicien, il te trouvera un douze pièces pour 2000 balles» / «les politiciens n'ont aucune idée de la dureté de la vie» / " est-ce que les politiciens savent» / « que les politiciens aillent voir comment ça se passe» / "les politiciens locaux, qualifiés de "médiocres" ».

17. Ainsi des jeunes dits «branchés» fournissent des clés explicatives; en prise avec le monde contemporain, ils sont modernes donc abstentionnistes : «Claude, 33 ans, directrice artistique dans l'édition... lit des magazines et regarde la télévision... La politique l'indiffère : elle n'a jamais eu de carte d'électeur, ne connait pas la date de l'élection présidentielle et ignore jusqu'à l'existence des législatives... Claude explique qu'elle connait les noms des principaux candidats, mais n'a pas envie d'en savoir plus : “L'autre jour, j'en ai entendu un, je ne sais plus lequel, qui parlait de façon un peu moins formatée que les autres. Mais au bout de cinq minutes, il s'est mis à dire du mal de ses concurrents, alors j’ai zappé...” » 
pas rare que s'exprime dans cette seconde vague de textes un certain retour vers des pratiques de mobilisation y compris conventionnelles, ce qu'attestent les propos d'un militant associatif se présentant dans la mouvance des listes citoyennes : «Je balancerai des idées subversives! Elles seront oubliées sur le moment, puis elles feront leur chemin et un jour on les ressortira... Dans cinq ans, 500 présidents de comité de quartier se présenteront. C'est comme cela que nous changerons les pratiques politiques. »

Que le rapport à la vie de la cité soit envisagé de façon moins négative qu'avant le 21 avril n'implique pas pour autant la valorisation du jeu politique. Il n'est cependant plus possible aux journalistes d'occulter les représentants institutionnels après le 21 avril; il leur faut dès lors parvenir à faire tenir ensemble le postulat de la distance au politique et la description de l'engagement citoyen. Les procédés qu'ils mobilisent méritent attention. C'est ainsi que, dans certains Carnets, des positions différentes sont assumées par plusieurs protagonistes, cette mise en scène permettant d'évoquer au sein d'un groupe posé comme existant en soi une palette différenciée de rapports au politique. Dans d'autres cas, si le journaliste veut que les opinions et réactions de certains groupes soient connues du lecteur, il lui faut bien passer par des intermédiaires institutionnels : ce sont les syndicalistes qu'il privilégie alors. Et quand il faut enfin en venir, dans un souci de réalisme, à évoquer des élus, leurs caractéristiques sociales sont particulièrement choisies : seuls sont habilités à prendre la parole des élus éminemment modestes, par exemple des militants syndicaux ou communistes de la France profonde ${ }^{18}$. On peut donc, là encore, vérifier la récurrence des raisonnements syllogistiques implicites que nous avions évoqués : le journalisme de proximité n'aborde les questions politiques, même dans le contexte exceptionnel d'une élection présidentielle dramatisée, qu'à partir d'une réception distanciée du jeu politique classique par des gens ordinaires.

C'est donc tant par une mise en récit du quotidien des individus ordinaires que par la restitution de leurs paroles qu'est lue en contrepoint la distance au politique, une coupure qui perdure dans l'ensemble des mises en récit en dépit du coup de tonnerre du 21 avril et des manifestations qui l'ont suivi. Rapporter des expériences individuelles permettrait non seulement de valider le propos, mais aussi d'expliquer la situation. Or, dans un Carnet et un seul, la correspondante locale du journal précise qu'un interviewé a refusé de dire son histoire personnelle :

18. On appréciera la virtuosité déployée pour décrire un sénateur-maire du Lot-et-Garonne présenté dans son rôle de modeste pompiste au service de l'intérêt général : "Depuis cinquantedeux ans, il tient la pompe à essence de Francescas. Le maire sait de quoi il parle. Il a plongé la main dans la glaise pendant cinquante ans de sa vie, avec son exploitation de 180 hectares. “Ça fait quinze ans que je veux prendre ma retraite ! s'écrie-t-il. Mais si je pompe plus l'essence, le marchand de journaux, l'épicerie et tout le reste vont s'en aller !" " 
«Avant, je n'existais pas, j'étais un numéro sur un dossier de la préfecture. Avec la lutte du collectif des sans-papiers, je suis sorti de l'ombre. Et plus jamais on ne m'y fera rentrer. » II veut qu'on l'appelle Mansour, mais ce n'est pas son vrai prénom. II est Algérien, a quitté son pays parce qu'il ne pouvait plus y vivre. "Je m'y sentais menacé », dit-il. C'est tout ce qu'il dira, parce qu'il ne veut pas entrer dans le détail de sa situation personnelle... "Ça n'a aucun intérêt, la question des sans-papiers est un problème global.»

Ces derniers propos soulèvent un problème de fond et remettent en question la pertinence même de ces reportages ${ }^{19}$ : refuser de se raconter c'est, fût-ce au prix de l'abandon de la sympathie immédiate du lecteur, imposer l'idée que seule la dimension collective, qui passe par une construction abstraite des faits sociaux, est à prendre en considération. C'est souligner que refuser le registre des particularismes pour privilégier l'énoncé général est la condition d'existence politique de ceux qui ne sont même pas reconnus comme citoyens. Ce que ce sans-papier suggère, c'est qu'individualiser, humaniser, rendre proche, équivaut à ôter aux questions sociales leur caractère d'universalité et les dépolitiser; une lecture des aspects strictement privés de sa situation dépouillerait celle-ci de sa dimension de problème public.

\section{L'imposition de sens ${ }^{20}$}

Ces mises en récit reposent sur le postulat de leur autosuffisance : les faits sociaux seraient transparents, les décrire permettrait de comprendre les situations et de les expliquer. Les procédés mobilisés par les journalistes, introspection et utilisation du discours rapporté, imposent indéniablement une clôture de sens en dépit de leur extrême artificialité.

\section{L'introspection, condition de la compréhension}

Pour faire comprendre des opinions et jugements provenant d'un univers social fréquemment éloigné de celui du lecteur, les journalistes en appellent aux affects. Nous en prendrons une illustration. Le texte du 21 mars d'A. Cojean, intitulé «On continue à parler de jeunes au lieu de dire voyous », permet de saisir par le rappel biographique les opinions d'un homme qui va voter Le Pen. Le registre relève du subjectif (la moitié des adjectifs utilisés appartiennent à cette classe) et de la dramatisation (avec une très forte fréquence de modalisation de négation). En atteste le début:

19. Paradoxe de l'exercice : c'est aussi un reportage qui nous le dit.

20. Nous reprenons ici l'idée de «significations préférentielles» des messages développée par D. Morley, 1992, "La réception des travaux sur la réception. Retour sur "Le public de Nationwide" ", Hermès, n 11-12. 
Comme il a aimé Jacques Chirac! Comme il a cru en lui ! «Une vraie passion », dit-il, se rappelant ses années de militantisme RPR, son engagement pour coller les affiches, distribuer les tracts... «J'aurais tout fait pour lui... » Bernard Burel ${ }^{21}$ parle d'une voix douce et ne masque pas ses émotions...

Ce sont les propos d'un homme passionné et blessé qui sont repris, ce que marquent la forte proportion de verbes déclaratifs et le large recours aux citations. Et le lecteur est pris dans le flot du discours tantôt cité, tantôt intégré, tantôt évoqué, un discours à la logique duquel il ne peut finalement échap$p^{22}$. Les évènements évoqués par le locuteur en disent plus long que des développements abstraits :

Une voiture est volée toutes les heures dans l'Oise... Il faudrait un plan de sauvetage de la France... ! Non, je ne suis pas raciste. Mais je souhaite qu'on stoppe l'immigration. Et je ne veux pas de mosquées. Nos racines appartiennent à l'Occident chrétien. Quiconque choisit la France doit se plier aux règles républicaines...

Une telle mise en forme ne peut que contraindre le lecteur à se mettre à la place de celui qu'il est invité à comprendre. Il ne s'agit plus là d'envisager de façon raisonnée les thèmes de l'extrême-droite, mais de suivre les cheminements d'un homme en chair et en os que tous les procédés stylistiques humanisent :

Alors, si Le Pen n'obtient pas ses 500 signatures, Burel le calme sortira de ses gonds et descendra dans la rue... «Et puis, ajoute-t-il, je me suiciderai politiquement. Et je déposerai dans l'urne un bulletin Jospin... » Juste avant de courir vers son coin de jardin où poussent des pétunias, des lobélias, des œillets d'Inde. Et une belle collection de fuchsias.

On ne saurait trop souligner combien ce type de récit, faisant entrer le lecteur de plain-pied dans un univers étranger, le déstabilise et le contraint à l'écoute empathique, procédé que maitrise particulièrement bien la grand reporter chevronnée qu'est $A$. Cojean, mais qui ne lui est pas spécifique ${ }^{23}$. La compétence autoréflexive des personnages est étonnante. Là réside sans doute l'ambigüité

21. Dont le nom est constamment martelé.

22. Sur le recours au style indirect libre, voir infra.

23. Ainsi Jean Eudes nous fait comprendre André, l'amour de la chasse et de la nature mais aussi le vote CPNT. La passion cynégétique va de soi tant elle s’inscrit dans la biographie («Pendant la guerre, nous étions douze enfants à la maison, et nous n'avions rien. Pour nous nourrir, mon père nous emmenait braconner...»); l'histoire personnelle permet de comprendre le vote ("Quand j'étais jeune, je votais pour de Gaulle, et je suis resté à droite. En 1995, j’ai voté Chirac dès le premier tour... La France doit soutenir M. Saint-Josse pour défendre la nature contre la directive Natura 2000 et contre les Verts : ces gens-là, on dirait qu'ils veulent s'approprier notre vie.»). Et, anticipant sur une éventuelle distanciation du lecteur, l'auteur rend le chasseur encore plus proche en évoquant son épouse ("C'était chaleureux la chasse, c'était notre but commun dans la vie»), assénant un argument définitif : "Avant de se mettre en ménage avec André, Paulette... a connu la vie d'usine et les luttes sociales : "Pendant longtemps, j'ai voté à gauche..."». 
la plus fondamentale de ces textes qui enserrent le lecteur dans les filets d'un fait social total, non seulement en mobilisant ses affects mais en déroulant simultanément, de façon linéaire et causale, une biographie très logiquement reconstruite. Si ces récits nous paraissent susceptibles de nous faire pénétrer le sens des actions humaines et, partant, le rapport au politique, c'est parce que tous obéissent à une logique de témoignage, celui-ci étant censé motiver un comportement électoral que le citoyen serait lui-même apte à expliquer. La proximité est ici transparence. C'est donc non seulement une logique de compréhension mais aussi une logique d'explication qui est développée, l'accumulation de récits de vie ou d'interactions dialogiques étant présentée comme ayant valeur de preuve. Le lecteur ne peut que s'en remettre au locuteur. Les profanes disposent de la compétence et de la lucidité indispensables pour donner à voir, donc analyser, la situation; c'est de leur expérience qu'émane le savoir $^{24}$. Impossible d'échapper aux clés de lecture proposées lorsque l'accumulation de problèmes concrets, chiffrés, permet de saisir le vote extrémiste au travers d'accidents biographiques ${ }^{25}$.

C'est encore et toujours du terrain qu'émanent après le 21 avril les analyses. Si, dans cette seconde séquence, la référence au politique s'impose, seule la valorisation de ce qui est présenté comme un rapport authentique à la chose publique est ici mise en avant, consacrant à nouveau l'existence d'une coupure radicale entre les préoccupations (cette fois reconnues comme politiques) de la base et les sommets de l'État. Tant au niveau des expériences associatives que sur le plan individuel, aucun des protagonistes mis en scène après le 21 avril ne méconnait les réalités de la cité et chacun est présenté comme étant le mieux à même d'apprécier les situations pour la simple raison qu'il les vit. Qu'on soit ancien trotskiste ou militant d'un parti classique, la vérité vient d'en bas, les analyses erronées d'en haut. Si cette seconde séquence redécouvre même des militants politiques, vétérans ou nouveaux adhérents, socialistes ou UDF, ceux-ci sont proches de la base, posent des questions essentielles, dénoncent le fossé entre élites dirigeantes et France d'en bas (eux/nous).

Chacun sait de quoi il parle, mais surtout chacun parle, grâce au journaliste, de ce dont ne savent pas parler les élus. C'est un leitmotiv :

Les hommes politiques sont dans un autre monde. Ils n'ont aucune idée de ce que c'est que de vivre avec le SMIC. Un patron... lui, il commence par mettre la main dans

24. Il faudrait citer dans son intégralité le récit portant sur la vie de Monsieur Lecru, gardien de prison retraité qui «sait de quoi il parle» (titré «Le Pen au premier tour, mais pas pour qu'il devienne président»).

25. Seul un lecteur particulièrement inhumain pourrait ne pas être touché par le témoignage de cet artisan victime de faillites successives ("Quand on est au fond du puits, on est prêt à tout pour remonter »). Dès la première phrase, l'accumulation de - bien pauvres - chiffres et l'invocation des besoins primaires du quotidien font appréhender le désespoir de la situation. 
le cambouis avec ses ouvriers. Qu'ils viennent donc passer une semaine avec un paysan! Ils n'ont plus de contact avec le réel, c'est pourquoi ils font des abus de pouvoir...26

Vécue et analysée par le bas, la mise en évidence de ce qu'il faut bien appeler une «fracture sociale » s'impose avec d'autant plus de force qu'elle émane de groupes exceptionnellement amenés à s'exprimer grâce au reportage. La réussite d'une telle mise en récit découle aussi d'une excellente connaissance de son lectorat par le journaliste, un lectorat dont il partage implicitement les présupposés et sait anticiper la capacité de déchiffrement.

\section{Le discours rapporté, clé de la proximité}

L'ensemble des Carnets repose sur l'utilisation des différentes palettes du discours rapporté 27 avec un large recours aux dialogues, procédés donnant vie aux échanges d'arguments. Nous ne pouvons, faute de place, examiner en détail la manière dont le rapporteur évalue l'énoncé pour l'intégrer, tantôt rapportant le mode de prise de parole, tantôt rapportant l'énoncé; bornons-nous à souligner combien ceci induit une impression de familiarité immédiate (c'est-à-dire sans intermédiaire apparent) avec les protagonistes des récits. Si l'ambigüité intrinsèque de ces procédés est bien connue ${ }^{28}$, force est pourtant de constater que le discours rapporté constitue le matériau privilégié de ces textes qui naviguent entre des citations fidèles mais rarement exhaustives et l'interprétation des propos censés être repris. L'illusion de transparence repose donc en fait sur l'art de l'intermédiaire, un journaliste narrateur dont la palette des prises de position va de l'effacement revendiqué (et plus ou moins abouti) à la construction assumée de la mise en scène.

Il faut d'ailleurs relever qu'après le 21 avril, les Carnets affichent un renforcement du caractère authentique des reportages puisque, à deux exceptions près, ce sont systématiquement les propos des interviewés qui sont repris entre guillemets dans les titres ${ }^{29}$. L'importance des citations, exceptionnellement élevée (en moyenne quinze par Carnet), la ponctuation exclamative ou

\section{4 mai 2002.}

27. Discours cité / intégré / narrativisé / évoqué.

28. On sait combien le discours rapporté, produisant des formes hybrides, fait débat chez les linguistes : voir P. Chareaudeau, D. Maingueneau, 2002, ouvr. cité, p. 195 et suiv.

29. 24 avril : «Après ça, les jeunes vont peut-être enfin s’intéresser à la politique ! ", 25 : « Le Pen au premier tour, mais pas pour qu'il devienne président», $26:$ «Si on m'avait dit que Le Pen passerait, je n'aurais pas voté Mamère », 27 : «Le PCF, c'est quand même un pan de l'histoire de France ! ", 30 : «C'est vrai que, parmi les jeunes, il y a aussi des fachos », 2 mai : "Le vote Le Pen, c'est l'électrochoc dont la France avait besoin », 3 : "Sur 75 électeurs du FN de ma commune, je dois en connaitre 30 », 4: «Agriculteurs, sinistrés : c'est pas une raison pour voter Le Pen ! ", 5: "Quand on est au fond du puits, on est prêt à tout pour remonter», 9: "Le seul régime démocratique, c'est le régime parlementaire !", $10:$ "Après le $1^{\text {er }}$ mai, il faut transformer l'émotion en action », 11 : «Voter à contrecœur pour un candidat, c'est ça la démocratie ? ", 
interrogative, la restitution d'un langage parlé, tout concourt à assoir la véracité, y compris la reprise des propos d'un interviewé qui « hésite à raconter son histoire dans le journal». Ces récits publiés en dernière page d'un quotidien dont le lectorat dispose majoritairement d'un fort capital culturel n'en paraissent-ils pas d'autant plus authentiques ? Une telle transparence est pourtant le fruit d'un savoir-faire paradoxal.

\section{Un exercice sous contrainte}

Après avoir souligné combien le Carnet repose sur la mise en exergue du particulier, il nous faut constater que la description débouche le plus fréquemment sur le général et ne peut le faire que parce qu'elle propose une représentation idéal-typique du social : groupes sociaux (notamment socioprofessionnels) et groupes d'opinion (existants ou présupposés par le journaliste). Une telle construction respecte en fait deux critères : un critère de diversité dans la prise en compte des catégories sociales, mais aussi un critère de représentativité.

Par le terme construction, nous ne signifions pas que le journaliste procède à la description de groupes qu'il constituerait de façon arbitraire, mais qu'il choisit de donner à voir et entendre des communautés variées, supposées pertinentes, et dont chacune serait homogène ${ }^{30}$. Sont montrés des groupes qui satisfont aux canons d'une sociologie spontanée fondée le plus souvent sur le postulat de l'homologie entre situations sociales et orientations politiques (les journalistes sacrifiant largement à une vision déterministe, en particulier celle de l'influence des variables lourdes : jeunes, chômeurs, agriculteurs... sont ainsi habilités à prendre la parole). Le choix des groupes repose sur leur recevabilité sociale ou plus exactement sur l'idée que s'en fait le journaliste : groupes d'individus dépolitisés dans la première séquence, groupes

15 : « Lionel Jospin n'a jamais su s'adresser aux petites gens», 16 : «Le vote, c'est une affaire sérieuse, je lirai les programmes», 18 : «En ville, les gens disent que c'est pourri ici, mais c'est faux ! », 21 : «Le 5 mai, j'ai donné des coups de trompette dans tout l'escalier», 22 : «Il faut secouer le PCF pour qu'il revienne réellement à gauche», $23:$ "Si je suis élu député, je balancerai des idées subversives ! », 24 : «Tous les jours on nous rappelle qu'on n'est pas d'ici ! », 25: «Union pour la majorité présidentielle ? Ça ne veut rien dire !», 28 : « Je pensais qu'on allait respecter le village martyr de Maillé», 29 : «Trop de gens mécontents cherchent des coupables ailleurs », 30 : «Le comportement de la gauche avec les harkis est dramatique ! », 31: « Agriculteurs pollueurs, les Verts nous ont insultés, ça ne s'oublie pas», $1^{\text {er }}$ juin : «Communiste, et s’il n'en reste qu'une, ce sera moi ! », $4:$ « Gouvernement de gauche ou pas, c'est les patrons qui font la loi », 5 : «Je suis régionaliste, je suis Bretonne avant d'être Française », 6 : "S'ils sont en prison, c'est qu'ils n'aiment pas la France ! », $7:$ "On ne vit pas en vase clos, on est citoyens avant d'être gays ! », 8 : «Je veux rouvrir la question du combat contre la drogue en France», 12 : «Le PS n'arrive pas à nous faire passer des messages clairs », 13 : «Après le premier tour, on a perdu notre principal bouc émissaire, Le Pen», 14 : "Le PC est complètement déconnecté des nouvelles réalités sociales», 15 : "Si on empêche les jeunes de s'exprimer, alors ils s'abstiennent ! », 18 : « Les réformes impopulaires vont enfin pouvoir être lancées ».

30. Lorsqu'il suppose que des groupes sociaux n'ont pas une unité de points de vue, on a vu qu'il en montre la diversité en la mettant en scène en un même lieu. 
d'individus socialisés dans le cadre de pratiques collectives à l'aune de la mobilisation postérieure au 21 avril. Représentativité et exotisme social seraient dès lors les deux critères principaux de sélection de ce que l'on peut considérer au final comme des études de cas légitimées par le contexte : désintérêt pour la politique au premier tour, mobilisation citoyenne au second. Le cadrage s'imposerait naturellement.

D'ailleurs, dans la deuxième séquence, alors que les récits de vie des interviewés sont moins développés au profit des argumentaires, les journalistes eux-mêmes donnent davantage à voir leur propre présence, suggérant qu'un espace d'échange se serait ouvert après le 21 avril et qu'il les impliquerait. Aussi vont-ils parfois jusqu'à indiquer qu'ils sont interpellés par leurs interviewés et qu'ils acceptent de faire remonter des messages. Il leur arrive aussi d'accentuer les procédés de construction, évoquant leur propre participation aux saynètes qu'ils mettent en récit. N'est-ce pas constater qu'avec la présence du FN au second tour des élections présidentielles, le seul cadrage socialement recevable est celui de la mobilisation citoyenne ? Mais le journaliste demeure le garant de la proximité, proximité avec la société civile d'en bas avant le 21 avril, proximité avec les espaces publics d'en bas après.

Comparer les deux séquences de Carnets confirme combien le choix de ce genre présuppose une évaluation particulière des rapports sociaux dont on a montré qu'elle perdure au fil du déroulement de la campagne, en dépit du «coup de tonnerre» du 21 avril. Le primat du social qui y est affirmé repose sur des procédés de construction maitrisés, il apparait comme un impératif catégorique qui consacre des valeurs à l'opposé de celles censées exister dans le champ politique traditionnel; en effet s'impose l'idée que les problèmes concrets, l'immédiateté de l'échange, l'absence de complexité, la vraie vie des vrais gens pourraient être lus et compris dans la transparence d'une simple restitution fidèle du quotidien. Or le quotidien qui est rapporté au lecteur du Monde est pour lui tout sauf quotidien, ce sont en fait des univers lointains, spécifiques, artificiellement mis en scène qui sont donnés non seulement à voir mais aussi à comprendre par le biais des affects et de l'introspection. C'est, dès lors, le caractère d'évidence de ce type de récit qu'il faut interroger. Ce n'est pas tant la recherche de proximité qui pose problème que les ressorts mobilisés pour imposer l'idée que le social pourrait être transparent et appréhendé par la seule description. Faire comme si l'économie de l'objectivation des faits sociaux était possible relève d'un classique bluff, non seulement littéraire et sociologique mais aussi politique ${ }^{31}$.

31. Ces Carnets ne constituent vraisemblablement qu'une déclinaison d'un phénomène plus général. Voir dans ce sens les analyses consacrées aux dispositifs télévisuels contemporains par G. Lochard et J.-C. Soulages, 2003 : "La parole politique à la télévision. Du logos à l'ethos », Réseaux, $\mathrm{n}^{\circ} 118$. 